SAÚDE E AMBIENTE

V.8 $\cdot$ N.1 Agosto/Setembro/Outubro - 2019

ISSN Digital: 2316-3798

ISSN Impresso: 2316-3313

DOI: 10.17564/2316-3798.2019v8n1p11-28 ORCID: 0000000000000
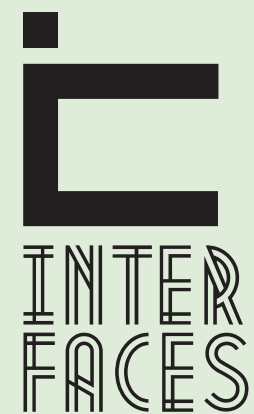

CIENTIFICAS

\section{ASPECTOS RELACIONADOS À INTERNAÇÃO HOSPITALAR EM ADULTOS PORTADORES DE HIV/AIDS ${ }^{1}$}

ASPECTS RELATED TOHOSPITAL ADMISSION IN ADULTS WITHHIV / AIDS

\section{ASPECTOS RELACIONADOS CON LA HOSPITALIZACIÓNEN} ADULTOS PORTADORES DE VIH / SIDA

1 Manuscrito originado da Dissertação de Mestrado de Cícera Eugênia Pereira da Silva, intitulada "Aspectos Relacionados À Internação Hospitalar Em Adultos Portadores De HIV/AIDS” defendida junto ao Programa de Pós-Graduação em Enfermagem da Universidade Federal de Sergipe em fevereiro de 2018.

\section{RESUMO}

Este trabalho teve por objetivo analisar aspectos relacionados à internação hospitalar em portadores de HIV/AIDS. Trata-se de um estudo transversal, realizado no Hospital Universitário de Aracaju/SE, no setor de infectologia. A amostra foi composta por 40 adultos HIV reagentes, dos quais $24(60,0 \%)$ eram homens, $20(50,0 \%)$ possuíam faixa etária abaixo de 40 anos e $27(67,5 \%)$ com menos de oito anos de estudo. Quanto à internação hospitalar, $31(77,5 \%)$ apresentaram diagnóstico tardio do HIV, com doenças relacionadas à AIDS, a exemplo de pneumonia $2(5,0 \%)$ e tuberculose 4 (10,0\%). Os dados deste estudo permitem afirmar que homens, população jovem e com pouca escolaridade são aspectos que corroboram para a internação hospitalar. A predominância entre os pacientes que apresentaram diagnóstico tardio é preocupante, principalmente por revelar falhas nos processos do cuidado à saúde.

\section{PALAVRAS-CHAVE}

Antígenos HIV, Síndrome de Imunodeficiência Adquirida, Hospitalização, Terapia Antirretroviral de Alta Atividade. 


\section{ABSTRACT}

This work aimed to analyze aspects related to hospital admission in adults with HIV/AIDS. This was a cross-sectional study, carried out at the University Hospital of Aracaju / SE, in the infectious sector. The sample consisted of 40 HIV-reactive adults. Of the 40 participants, 24 (60.0\%) were men, 20 (50.0 $\%)$ had an age group below 40 years and 27 (67.5\%) with less than eight years of study. As to hospital admission, $31(77.5 \%)$ had a late diagnosis of HIV, with AIDS-related diseases, such as pneumonia $2(5.0 \%)$ and tuberculosis $4(10.0 \%)$. The data of this study allow to affirm that men, young population and with little schooling are aspects that corroborate for the hospitalization. The predominance among the patients who presented a late diagnosis is worrisome, mainly because it reveals flaws in the health care processes.

\section{KEYWORDS}

HIV antigens, Acquired Immunodeficiency Syndrome, Hospitalization, High Activity Antiretroviral Therapy.

\section{RESUMEN}

Este trabajo tuvo como objetivo analizar aspectos relacionados a la internación hospitalaria en portadores de VIH/SIDA. Se trata de un estudio transversal, realizado en el Hospital Universitario de Aracaju / SE, en el sector de infectología. La muestra fue compuesta por 40 adultos VIH reactivos. De los 40 participantes, 24 (60,0\%) eran hombres, 20 (50,0\%) tenían rango de edad por debajo de 40 años y 27 (67,5\%) con menos de ocho años de estudio. En cuanto a la internación hospitalaria 31 $(77,5 \%)$ presentaron diagnóstico tardío del $\mathrm{VIH}$, con enfermedades relacionadas al sida, a ejemplo de neumonía 2 (5,0\%) y tuberculosis 4 (10,0\%). Los datos de este estudio permiten afirmar que hombres, población joven y con poca escolaridad son aspectos que corroboran para la internación hospitalaria. El predominio entre los pacientes que presentaron diagnóstico tardío es preocupante, principalmente por revelar fallas en los procesos del cuidado a la salud.

\section{PALABRAS-CLAVE}

Antígenos VIH, Síndrome de Inmunodeficiencia Adquirida, Hospitalización, Terapia Antirretroviral de Alta Actividad. 


\section{INTRODUÇãO}

A AIDS (Síndrome da Imunodeficiência Humana) configura-se, atualmente, como um relevante problema de saúde pública mundial. Dados advindos do relatório do Programa Conjunto das Nações Unidas sobre HIV/AIDS (UNAIDS) confirmam que, no mundo, há 36,7 milhões de pessoas vivendo com o Vírus da Imunodeficiência Humana - HIV (UNAIDS, 2016).

No Brasil, do início da epidemia da AIDS, até junho de 2016, foram registrados 842.710 casos, destes, $65,1 \%$ ocorreram em homens. Em relação ao coeficiente de mortalidade no país, observou- se um discreto declínio passando de 5,9\% em 2006 para 5,6\% em 2015. No estado de Sergipe, a incidência em 2015 correspondeu a 17,1 por 100.000/ habitantes (BRASIL, 2016).

No país, após 30 anos do primeiro caso de AIDS em 1981, a política de enfrentamento à epidemia é reconhecida como referência no âmbito internacional (GRECO, 2016). Em dezembro de 2013, o Brasil tornou- se o primeiro país em desenvolvimento e o terceiro do mundo, a iniciar a terapia com antirretrovirais (TARV) para as pessoas acometidas pelo HIV/AIDS, independente do aparecimento da imunodepressão. Em 2014, o Ministério da Saúde (MS) recomendou o esquema de primeira linha para o tratamento constituído por três fármacos em um só comprimido: tenofovir + lamivudina + efavirenz (TDF + 3TC + EFV) (BRASIL, 2015).

Porém, em 2017, um outro medicamento foi incorporado ao manejo terapêutico, o dolutegravir (DTG). Dessa forma, o novo esquema para início de tratamento para pessoas vivendo com HIV/AIDS (PVHA) passou a ser constituído por: TDF+ 3TC+ DTG (BRASIL, 2017).

Um grande desafio no tratamento da AIDS para as políticas públicas de saúde está relacionado à adesão. Para se alcançar a eficácia do tratamento e a redução da replicação viral do HIV, é necessária adesão aos esquemas terapêuticos superior a 80,0\% (SILVA et al., 2015). 0 risco de transmissão da doença está intrinsicamente relacionado à falta de adesão ao tratamento, por isso, faz-se necessário o acompanhamento e uma assistência à saúde individualizada (HIPOLITO et al., 2017).

Não obstante aos avanços da terapia antirretroviral e o aumento na sobrevida dos pacientes infectados pelo HIV, um grande número de pessoas pertencentes a esse grupo ainda necessita de hospitalização. Algumas situações são apontadas como predisponentes à internação, tais como: sexo masculino, população jovem, nível socioeconômico precário, uso de drogas ilícitas e desenvolvimento de doenças oportunistas (NUNES et al., 2015).

Diante do exposto, o objetivo deste estudo foi analisar os aspectos relacionados à internação hospitalar em adultos portadores de HIV/AIDS.

\section{MÉTOdOS}

A abordagem metodológica foi constituída por um estudo transversal com descrição de variáveis e análise de aspectos relacionados às internações hospitalares, entre as pessoas vivendo com HIV/AIDS. 
A pesquisa foi realizada no Hospital Universitário (HU) de Aracaju/SE, no setor de internamento da infectologia, onde funciona a clínica médica II. Essa unidade possui 18 leitos, desses, onze são destinados às doenças infecciosas com uma média de ocupação de $\mathbf{7 4 , 9 1 \%}$ (SILVA et al., 2016).

Utilizou- se a amostra intencional, composta por 40 pessoas e os critérios de inclusão dos participantes foram: pessoas do sexo masculino e feminino com idade maior ou igual a 18 anos, alfabetizados ou não; comprovadamente HIV positivo que estiveram internados no Hospital Universitário de Aracaju, entre o período de dezembro de 2016 a outubro de 2017. Foram excluídos os pacientes que demonstravam não ter conhecimento sobre o diagnóstico de HIV positivo e também aqueles que já haviam respondidos ao questionário anteriormente.

Para a realização da entrevista aos pacientes, aplicou- se um formulário contendo informações sobre as seguintes variáveis: identificação, dados sóciodemográficos; epidemiológicos comportamentais/apoio; terapia antirretroviral, adesão e dados da internação. Para obtenção de alguns dados complementares como: tratamento medicamentoso prescrito, exames laboratoriais (carga viral e $C D 4$ ) e outros exames que se fizeram pertinentes ao objeto do estudo, utilizou-se informações contidas nos prontuários. Além destes, buscou- se os profissionais de saúde e algumas vezes o representante legal, nos casos nos quais o paciente apresentou alguma condição clínica que o impossibilitasse em responder as questões.

Durante o período da coleta, os pacientes foram identificados pelo censo de admissão da unidade selecionada. Após serem observados os critérios de exclusão, buscava- se um ambiente privativo para realização da entrevista.

Foram respeitados todos os aspectos contidos na Resolução 466/2012 do Comitê Nacional de Ética e Pesquisa. O projeto foi aprovado pelo Comitê de Ética em Pesquisa da Universidade Federal de Sergipe sob o número de parecer 1.849.121 e CAAE 57993616.6.0000.5546.

Os dados foram descritos por meio de frequências simples e percentuais. Para avaliar as relações entre as características demográficas, econômicas e comportamentais referentes à análise dos aspectos da TARV e de internação, foi aplicado a análise de correspondência múltipla para construção de um mapa perceptual. Dessa forma, foram aplicados testes exatos de Fisher para todos os pares de variáveis categóricas entre os grupos. Todos os pares de variáveis com associação ao nível de 5,0\% foram incluídas no citado mapa (GREENACRE; BLASIOS, 2006). 0 nível de significância adotado foi de $5,0 \%$ e o software utilizado foi o $R$ Core Team 2017.

\section{RESULTADOS}

Dos 40 pacientes entrevistados, 24 (60,0\%) eram do sexo masculino, (50,0\%), com Intervalo de Confiança (IC) (45,0-75,0), encontravam-se na faixa etária abaixo de 40 anos sendo o IC (35,0$65,0)$, mais que a metade $(21 / 52,5 \%)$ possuíam renda e $27(67,5 \%)$ tinham menos que oito anos de estudo, IC $(52,5-82,5)$. Quanto a cor autorreferida, a maioria era parda $(25 / 62,5 \%)$ e residia na grande Aracaju (22/55,0\%) (Tabela 1). 
Tabela 1- Caracterização dos aspectos demográficos e econômicos da amostra. Período: outubro de 2016 a novembro de 2017. Aracaju/SE.

\begin{tabular}{|c|c|c|c|}
\hline Aspectos demográficos & n (40) & $\%$ & *IC 95\% \\
\hline \multicolumn{4}{|l|}{ Sexo } \\
\hline Feminino & 16 & $40,0 \%$ & $25,0-55,0$ \\
\hline Masculino & 24 & $60,0 \%$ & $45,0-75,0$ \\
\hline \multicolumn{4}{|l|}{ Faixa Etária } \\
\hline$<=40$ anos & 20 & $50,0 \%$ & $35,0-65,0$ \\
\hline$>40$ anos & 20 & $50,0 \%$ & $35,0-65,0$ \\
\hline \multicolumn{4}{|l|}{ Renda } \\
\hline Não possui renda & 19 & $47,5 \%$ & $32,5-65,0$ \\
\hline Possui renda & 21 & $52,5 \%$ & $35,0-67,5$ \\
\hline \multicolumn{4}{|l|}{ Escolaridade } \\
\hline$<=8$ anos & 27 & $67,5 \%$ & $52,5-82,5$ \\
\hline$>8$ anos & 13 & $32,5 \%$ & $17,5-47,5$ \\
\hline \multicolumn{4}{|l|}{ Cor Autorreferida } \\
\hline Parda & 25 & $62,5 \%$ & $47,5-77,5$ \\
\hline Não parda & 15 & $37,5 \%$ & $22,5-52,5$ \\
\hline \multicolumn{4}{|l|}{ Local da Residência } \\
\hline **Grande Aracaju & 22 & $55,0 \%$ & $40,0-70,0$ \\
\hline Demais municípios do Estado & 18 & $45,0 \%$ & $30,0-60,0$ \\
\hline
\end{tabular}

*IC 95\% - Intervalo com 95\% de confiança via bootstrap (1000 réplicas). **Inclui: Aracaju, São Cristóvão, Nossa Senhora do Socorro, Barra dos Coqueiros

Fonte: Dados da pesquisa

Com relação aos aspectos epidemiológicos e comportamentais que levaram os pacientes à internação, 21 (52,5\%) relataram não possuir parceria fixa, 23 (59,0\%) tiveram um ou mais parceiros fixos no último ano. Dos participantes da pesquisa, apenas 29 souberam responder como adquiriram o vírus, destes $24(82,8 \%)$ mencionaram transmissão do HIV por via sexual. Atinente à orientação sexual $35(87,5 \%)$ relataram heterossexualidade, com IC $(75,0$ - 97,5) e aproximadamente um terço deles verbalizaram não fumar, usar drogas ilícitas ou bebidas alcoólicas. Quanto ao tempo de descoberta do HIV, 18 (45,0\%) afirmaram saber de sua condição há mais de cinco anos (Tabela 2). 
Tabela 2 -Aspectos epidemiológicos e comportamentais que levaram à internação hospitalar. Período: outubro de 2016 a novembro de 2017. Aracaju/SE.

\begin{tabular}{|c|c|c|c|}
\hline Aspectos epidemiológicos e comportamentais & $\mathbf{n}$ & $\%$ & *IC 95\% \\
\hline \multicolumn{4}{|l|}{ Parceiro Fixo $(n=40)$} \\
\hline Sim & 19 & $47,5 \%$ & $32,5-65,0$ \\
\hline Não & 21 & $52,5 \%$ & $35,0-67,5$ \\
\hline \multicolumn{4}{|l|}{ Transmissão do HIV (n=29) } \\
\hline Sexual & 24 & $82,8 \%$ & $69,0-96,6$ \\
\hline Transfusão de Sangue & 2 & $6,9 \%$ & $0,0-17,2$ \\
\hline Uso de drogas injetáveis & 1 & $3,4 \%$ & $0,0-10,3$ \\
\hline Perfurocortante & 2 & $6,9 \%$ & $0,0-17,2$ \\
\hline \multicolumn{4}{|l|}{ Etilismo $(n=40)$} \\
\hline Sim & 11 & $27,5 \%$ & $13,8-48,3$ \\
\hline Não & 29 & $72,5 \%$ & $51,7-86,2$ \\
\hline \multicolumn{4}{|l|}{ Tabagismo $(n=39)$} \\
\hline Sim & 9 & $23,1 \%$ & $10,3-35,9$ \\
\hline Não & 30 & $76,9 \%$ & $64,1-89,7$ \\
\hline \multicolumn{4}{|l|}{ Droga Ilícita $(n=40)$} \\
\hline Sim & 11 & $27,5 \%$ & $15,0-42,5$ \\
\hline Não & 29 & $72,5 \%$ & $57,5-85,0$ \\
\hline \multicolumn{4}{|l|}{ Tempo de descoberta da doença $(n=40)$} \\
\hline$<1$ ano & 10 & $25,0 \%$ & $12,5-40,0$ \\
\hline 1 a 5 anos & 12 & $30,0 \%$ & $17,5-44,9$ \\
\hline$>5$ anos & 18 & $45,0 \%$ & $30,0-60,0$ \\
\hline \multicolumn{4}{|l|}{ Orientação sexual $(n=40)$} \\
\hline Heterossexual & 35 & $87,5 \%$ & $75,0-97,5$ \\
\hline $\mathrm{HSH}^{* *}$ & 1 & $2,5 \%$ & $0,0-7,5$ \\
\hline MSM*** & 0 & $0,0 \%$ & $0,0-0,0$ \\
\hline Bissexual & 4 & $10,0 \%$ & $2,5-20,0$ \\
\hline
\end{tabular}




\begin{tabular}{|c|c|c|c|}
\hline Aspectos epidemiológicos e comportamentais & $\mathbf{n}$ & $\mathbf{\%}$ & *IC 95\% \\
\hline Travesti & 0 & $0,0 \%$ & $0,0-0,0$ \\
\hline Transsexual/Transgênero & 0 & $0,0 \%$ & $0,0-0,0$ \\
\hline Node parceiros fixos no último ano $(\mathrm{n}=39)$ & & & \\
\hline$>=1$ & 23 & $59,0 \%$ & $43,6-74,4$ \\
\hline Nenhum & 16 & $41,0 \%$ & $25,6-56,4$ \\
\hline
\end{tabular}

*IC 95\% - Intervalo com 95\% de confiança via bootstrap (1000 réplicas). **HSH: Homem que têm relações sexuais com outros homens. ***MSM: Mulheres que têm relações sexuais com outras mulheres. Fonte: Dados da pesquisa

$\mathrm{Na}$ identificação sobre uso da terapia antirretroviral, 24 (70,6\%) dos pacientes relataram ter iniciado os medicamentos com menos de 30 dias após o diagnóstico o IC correspondeu a $(55,9-85,3)$. Ao questionar sobre efeitos colaterais dos medicamentos, 19 (47,5\%) referiram apresentar e os mais citados foram: náuseas, vômitos, diarreia e insônia.Com relação ao comparecimento às consultas, 12 $(69,7 \%)$ compareciam com frequência aos agendamentos (Tabela 3$)$.

Tabela 3 - Distribuição das informações sobre uso da Terapia antirretroviral (TARV). Período: outubro de 2016 a novembro de 2017. Aracaju/SE.

\begin{tabular}{|c|c|c|c|}
\hline Informações sobre TARV & N & \% & *IC 95\% \\
\hline Uso do TARV após diagnóstico de HIV $(\mathrm{n}=34)$ & & & \\
\hline$<=30$ dias & 24 & $70,6 \%$ & $55,9-85,3$ \\
\hline$>30$ dias & 10 & $29,4 \%$ & $14,7-44,1$ \\
\hline Efeitos colaterais aos TARV $(\mathrm{n}=40)$ & & & \\
\hline Diarreia & & & \\
\hline Sim & 4 & $33,3 \%$ & $8,3-58,3$ \\
\hline Não & 8 & $66,7 \%$ & $41,7-91,7$ \\
\hline Vômito & & & \\
\hline Sim & 3 & $25,0 \%$ & $0,0-50,0$ \\
\hline Não & 9 & $75,0 \%$ & $50,0-100,0$ \\
\hline Náuseas & & & \\
\hline Sim & 4 & $36,4 \%$ & $9,1-63,6$ \\
\hline Não & 7 & 63,6 & $36,4-90,9$ \\
\hline
\end{tabular}




\begin{tabular}{|c|c|c|c|}
\hline Informações sobre TARV & $\mathbf{N}$ & $\%$ & *IC 95\% \\
\hline \multicolumn{4}{|l|}{ Manchas Vermelhas } \\
\hline Sim & 1 & $8,3 \%$ & $0,0-25,0$ \\
\hline Não & 11 & $91,7 \%$ & $75,0-100,0$ \\
\hline \multicolumn{4}{|l|}{ Agitação } \\
\hline Sim & 1 & $8,3 \%$ & $0,0-25,0$ \\
\hline Não & 11 & 91,7 & $75,0-100,0$ \\
\hline \multicolumn{4}{|l|}{ Insônia } \\
\hline Sim & 3 & $25,0 \%$ & $0,0-50,0$ \\
\hline Não & 9 & $75,0 \%$ & $50,0-100,0$ \\
\hline \multicolumn{4}{|l|}{ Outros } \\
\hline Sim & 3 & $25,0 \%$ & $0,0-50,0$ \\
\hline Não & 9 & $75,0 \%$ & $50,0-100,0$ \\
\hline Sem sintomas & 28 & $70,0 \%$ & $55,0-85,0$ \\
\hline \multicolumn{4}{|c|}{ Comparecimento às consultas agendadas $(n=40)$} \\
\hline Sempre & 23 & $57,3 \%$ & $42,5-72,5$ \\
\hline Às vezes & 7 & $17,5 \%$ & $7,5-30,0$ \\
\hline Nunca & 3 & $7,5 \%$ & $0,0-17,5$ \\
\hline Não se aplica & 7 & $17,5 \%$ & $7,5-30,0$ \\
\hline
\end{tabular}

*IC 95\% - Intervalo com 95\% de confiança via bootstrap (1000 réplicas). Fonte: Dados da pesquisa

Nos aspectos relacionados à internação hospitalar, 31 (77,5\%) dos pacientes tiveram menos que um ano entre o diagnóstico e a primeira internação, 10 (25,0\%) referiram pelo menos uma internação no último ano e 24 (60,0\%) não necessitaram de internação. Quanto aos motivos clínicos, 24 (60,0\%) afirmaram sentir fraqueza, diarreia e febre (15/40,5\%), anemia $(11 / 27,5 \%)$ entre outros. Em se tratando de outros diagnósticos relacionados à AIDS, 2 (5,0\%) apresentaram pneumonia, 4 (10,0\%) tuberculose e $4(10,0 \%)$ toxoplasmose cerebral, entre outros (Tabela 4$)$. 
Tabela 4 - Aspectos relacionados à internação hospitalar. Período: outubro de 2016 a novembro de 2017. Aracaju/SE.

\begin{tabular}{|c|c|c|c|}
\hline Informações sobre internação hospitalar & $n(40)$ & $\%$ & *IC 95\% \\
\hline \multicolumn{4}{|c|}{ Tempo entre o diagnóstico e a primeira internação } \\
\hline$<=1$ ano & 31 & $77,5 \%$ & $62,5-90,0$ \\
\hline$>1$ ano & 9 & $22,5 \%$ & $10,0-37,5$ \\
\hline \multicolumn{4}{|l|}{ Dados clínicos da internação } \\
\hline Anemia & 11 & $27,5 \%$ & $16,2-45,9$ \\
\hline Fraqueza & 24 & $60,0 \%$ & $48,6-78,4$ \\
\hline Diarreia & 15 & $40,5 \%$ & $27,0-56,8$ \\
\hline Febre & 15 & $40,5 \%$ & $24,3-56,8$ \\
\hline Caquexia & 10 & $27,0 \%$ & $13,5-40,5$ \\
\hline Lesão pele Mucosa & 9 & $24,3 \%$ & $10,8-37,8$ \\
\hline Alteração Sistema Nervoso Central & 8 & $21,6 \%$ & $10,8-35,1$ \\
\hline Não se aplica & 24 & $60,0 \%$ & $13,5-40,5$ \\
\hline \multicolumn{4}{|l|}{ Outros diagnósticos relacionados à AIDS } \\
\hline \multicolumn{4}{|l|}{ Pneumonia } \\
\hline Sim & 2 & $5,0 \%$ & $0,0-12,5$ \\
\hline Não & 38 & $95,0 \%$ & $87,5-100,0$ \\
\hline \multicolumn{4}{|l|}{ Tuberculose } \\
\hline Sim & 4 & $10,0 \%$ & $2,5-20,0$ \\
\hline Não & 36 & $90,0 \%$ & $80,0-97,5$ \\
\hline \multicolumn{4}{|l|}{ Herpes Simples } \\
\hline Sim & 2 & $5,0 \%$ & $0,0-12,5$ \\
\hline Não & 38 & 95,0 & $87,5-100,0$ \\
\hline \multicolumn{4}{|l|}{ Citomegalovírus } \\
\hline Sim & 2 & $5,0 \%$ & $0,0-12,5$ \\
\hline Não & 38 & $95,0 \%$ & $87,5-100,0$ \\
\hline \multicolumn{4}{|l|}{ Candidíase de Esôfago } \\
\hline Sim & 3 & $7,5 \%$ & $0,0-17,5$ \\
\hline
\end{tabular}




\begin{tabular}{|c|c|c|c|}
\hline Informações sobre internação hospitalar & $\mathbf{n ( 4 0 )}$ & $\mathbf{\%}$ & *IC 95\% \\
\hline Não & 37 & $92,5 \%$ & $82,5-100,0$ \\
\hline Toxoplasmose Cerebral & & & \\
\hline Sim & 4 & $10,0 \%$ & $2,5-20,0$ \\
\hline Não & 36 & $90,05 \%$ & $80,0-97,5$ \\
\hline Outras & & & \\
\hline Sim & 6 & $15,0 \%$ & $5,0-27,5$ \\
\hline Não & 34 & $85,0 \%$ & $72,5-95,0$ \\
\hline 1 vez & 10 & $25,0 \%$ & $12,5-40,0$ \\
\hline 2 vezes & 4 & $10,0 \%$ & $0,0-20,0$ \\
\hline 3 vezes & 1 & $2,5 \%$ & $0,0-7,5$ \\
\hline Mais de 3 vezes & 1 & $60,0 \%$ & $45,0-75,0$ \\
\hline Não se aplica & & & $0,0-7,5$ \\
\hline
\end{tabular}

*IC 95\% - Intervalo com 95\% de confiança via bootstrap (1000 réplicas). Fonte: Dados da pesquisa

Em relação ao mapa perceptual, observando as categorias e seus quadrantes, identifica- se quatro perfis: Perfil 1: Pacientes com menos de 40 anos, que sabem da doença entre um a menos de 5 anos, que possuem outros diagnósticos relacionados a aids e fazem uso de bebida alcoólica. Perfil 2: Pacientes que não foram internados por diarreia ou alteração do sistema nervoso central, que não responderam sobre como adquiriram o vírus, que outro motivo levou a internação e fumam. Perfil 3: Pacientes com mais de 40 anos, que sabem da doença há mais de 5 anos, não fazem uso de bebida alcoólica, não tem outros diagnósticos associados a aids e não tem reação ao fazer uso desses medicamentos. Perfil 4: Pacientes que se internaram devido a alteração do sistema nervoso central ou diarreia e nenhuma outra causa, não fumam, tem reação ao fazer uso desses medicamentos e responderam como adquiriram o vírus (Figura 1). 
Figura 1 - Mapa Perceptual relacionado aos aspectos da internação hospitalar. Período: outubro de 2016 a novembro de 2017. Aracaju/SE

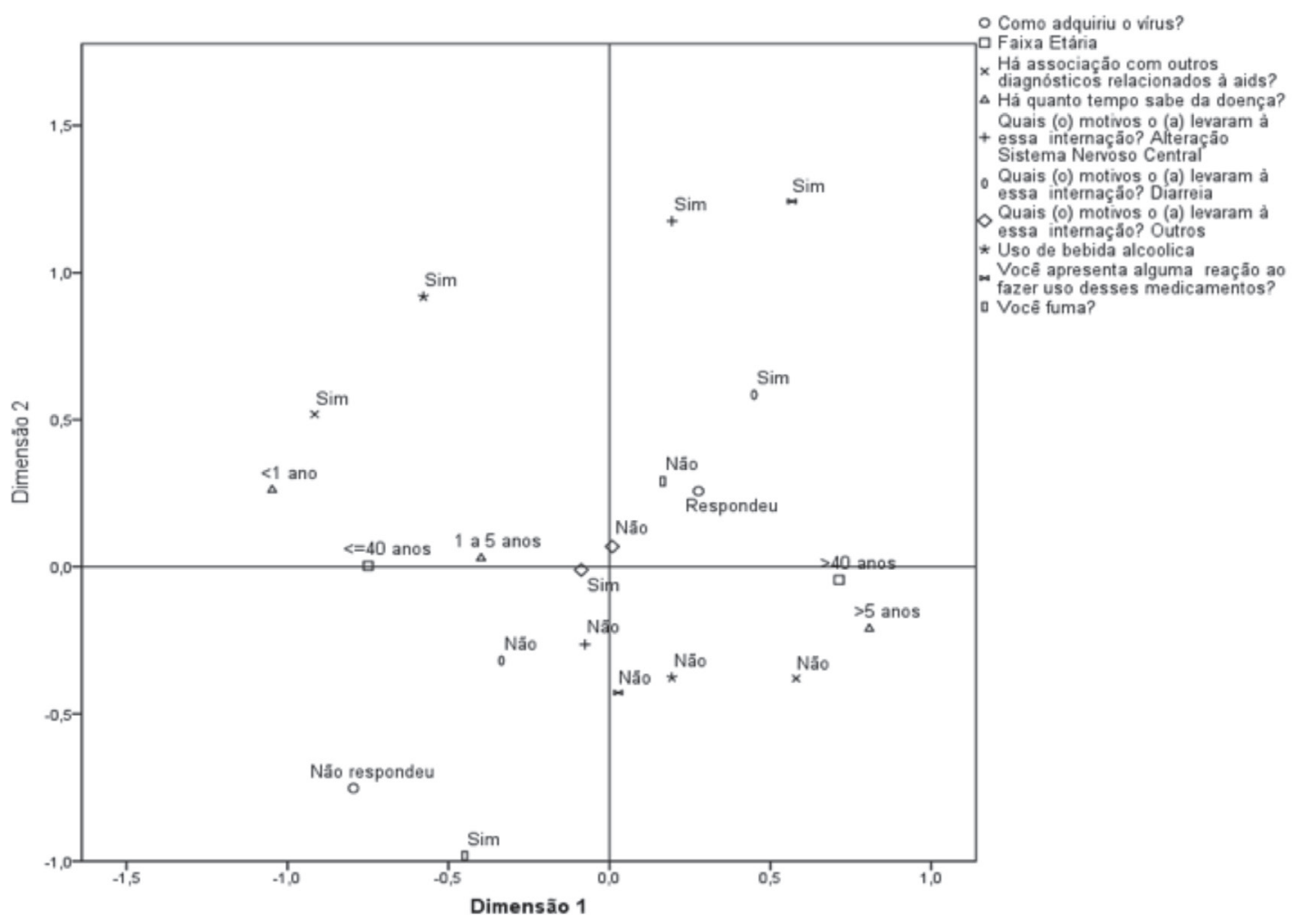

\section{DISCUSSÃO}

O acesso universal ao tratamento, à prevenção da infecção pelo HIV e das demais infecções sexualmente transmissíveis possibilitou transformar o cenário da epidemia do HIV no país. No entanto, os achados deste estudo revelam que a hospitalização relacionada ao HIV no Hospital Universitário de Sergipe foi elevada, principalmente, ao se considerar 21 anos após a introdução da terapia antirretroviral no Brasil (BRASIL, 2008). Por outro lado, houve um predomínio entre os indivíduos que não necessitaram de novas internações no último ano, o que se pode inferir uma maior sensibilização para adesão, após a experiência da primeira internação (SILVA et al., 2015).

Fato mais preocupante ainda encontrado é que mais de setenta por cento dos pacientes com menos de um ano de diagnóstico foram internados já com sinais e sintomas relacionados à AIDS. Esses 
dados contrapõem ao fato dos pacientes, em sua grande maioria, terem iniciados a terapia antirretroviral em pelo menos trinta dias após o diagnóstico, o que remete à reflexão sobre o diagnóstico tardio desses pacientes, pois mesmo tendo iniciado o TARV, ainda necessitaram de internação. Este achado pode estar relacionado com a falha terapêutica, principalmente pela não-adesão do paciente à essa terapia (FIGUEIREDO et al., 2006). No estado de Sergipe, vem ocorrendo diversas campanhas para detectar o vírus por meio da realização do teste de imunoensaio (IE) simples, também denominado pelo MS de teste rápido. Porém, há necessidade de mais informações a respeito da temática para sensibilizar a população, sobretudo, a respeito da prevenção (MÉRICI, 2016).

Dentre os dados clínicos que levaram à última internação, fraqueza se constituiu o mais citado, seguido de diarreia, febre e anemia. Esses achados do estudo convergem com o de Kumar et al. (2007), que demonstraram estas sintomatologias como as possíveis causas de internações. Neste estudo, dentre outros diagnósticos relacionados à AIDS constatou- se uma predominância entre as doenças infecciosas como pneumonia, tuberculose, toxoplasmose cerebral. Apesar do advento da Terapia Antirretroviral Altamente Eficaz (HAART), as doenças oportunistas ainda são as principais responsáveis pelas internações hospitalares (NUNES et al., 2015).

Sergipe, como em todos os estados do Brasil, fornece a terapia antirretroviral combinada a todos portadores elegíveis de HIV/AIDS, desde 1996, garantidos pela lei nº 9.313 sancionada em 13 de novembro, que assegura aos portadores do HIV e AIDS o direito de receber gratuitamente, pelo Sistema Único de Saúde (SUS), todos os medicamentos necessários ao seu tratamento (BRASIL, 2006). O acesso ao TARV, associado ao uso de quimioprofilaxia para infecções oportunistas e a oferta de outros tipos de assistência, tem possibilitado a redução das internações hospitalares e dos óbitos por AIDS no Brasil (BRASIL, 2008).

A política brasileira de acesso universal à TARV alcançou bons resultados, como diminuição da morbimortalidade, redução de internações e dos custos do tratamento (BARROS; SILVA, 2017). Para continuidade e avanço desse sucesso, elevadas taxas de adesão necessitam ser mantidas em todo o país. Com isso, além de assegurar o acesso ao tratamento, é necessário melhorar a qualidade do cuidado, também investir nas políticas que atenuam os problemas sociais e culturais que afetam os pacientes vivendo com HIV/AIDS (MELCHIOR et al., 2007).

Dados do MS mostram que algum grau de não-adesão ocorre universalmente, tanto em países ricos como em países pobres, ocorrendo mesmo em doenças que envolvem potenciais riscos de vida. Os pacientes têm "momentos de não-adesão" e a história dos que têm boa adesão tem relação com a superação de dificuldades (BRASIL, 2010).

Os resultados desta pesquisa revelam uma população predominantemente masculina, similar aos dados nacionais e das macrorregiões do país (NUNES et al., 2015). Nota-se que os homens frequentemente não se atentam para o autocuidado, com uma busca reduzida pela assistência à saúde. Esse fato possivelmente pode estar relacionado ao maior número de internação para esse gênero.

No Brasil, constatou- se um incremento no número de pessoas jovens, principalmente entre àquelas compreendidas na faixa etária de 15 a 24 anos, conforme mencionado no último boletim epidemiológico publicado pelo Ministério da Saúde em 2016. É preocupante essa realidade, pois são 
pessoas que se encontram no auge da sua produtividade e de descobertas das potencialidades. Este estudo evidenciou que metade dos entrevistados possuía menos de quarenta anos, o que reflete uma realidade semelhante aos dados apresentados pelo MS (BRASIL, 2016).

Em relação à renda, sabe- se que uma remuneração digna possibilita um tratamento adequado que atenda às necessidades holísticas dos pacientes, propiciando dessa forma uma vida mais saudável. Porém, o estudo verificou que apesar da maioria relatar possuir renda, muitos desses detinham um poder aquisitivo inferior a um salário mínimo. No que tange aos anos de estudo, equiparando aos índices nacionais, a pesquisa mostrou que os participantes possuíam poucos anos de escolaridade. Esse fato reflete no menor acesso à informação o que dificulta a apreensão sobre adesão ao tratamento e sua importância para uma melhor qualidade de vida (BRASIL, 2010).

A cor autorreferida, por ser uma impressão subjetiva, é uma caracterização de difícil análise, tanto pela população ser resultante de miscigenações, quanto por representar a forma que se é enxergado. No entanto, a cor parda foi preponderante neste estudo, o que corrobora com os dados apresentados pelo MS (BRASIL, 2016).

Verificou- se que o local de residência dos participantes de maior prevalência foi a grande Aracaju, na qual concentrou, outras três cidades, além de Aracajú: Nossa Senhora do Socorro, São Cristóvão e Barra dos Coqueiros. Observa- se, no entanto, uma tendência nacional para a interiorização do HIV, realidade essa encontrada no estudo realizado por Pedrosa et al. (2015). Pode- se depreender que uma vez residindo no interior do estado, haja uma dificuldade maior para ter acesso às consultas ambulatoriais realizadas nos centros de referência. Não havendo a continuidade da assistência pode ocorrer consequentemente, a necessidade de um maior número de internamentos.

Em se tratando de relacionamentos afetivos muitos deles relataram desinteresse em manter um parceiro (a). Talvez pelo receio de não serem aceitos ao relatar o diagnóstico do HIV, muitos dos entrevistados informaram optar por não assumir um relacionamento estável. Sabe- se que as boas relações pessoais impulsionam as pessoas a buscar uma vida mais prazerosa e feliz, dessa maneira, estabelecer laços afetivos torna- se primordial para o enfrentamento da doença (SILVA et al., 2016).

No Nordeste prevalece a transmissão sexual, semelhante aos dados nacionais. Quanto à orientação sexual, ocorreu um predomínio para categoria heterossexual, o que conjuga com os dados apresentados no último boletim do MS. Porém, observa- se um recrudescimento entre os homens que fazem sexo com homens (HSH) (BRASIL, 2016). Durante a entrevista pôde- se observar um desconforto ao responder esse questionamento, por se tratar de algo íntimo o que, possivelmente, a informação relacionada a orientação sexual, pode não corresponder totalmente à realidade.

O uso das drogas lícitas e ilícitas podem levar ao insucesso do tratamento e a possíveis novas internações. Há estudos nos quais os pacientes mencionam ter que parar momentaneamente o uso dos medicamentos para ingerirem bebida alcoólica (ROMEU et al., 2012). Nesta pesquisa, mesmo havendo o relato, que no momento não faziam mais uso, muitos deles haviam citados o contato assíduo com essas drogas, tendo cessado ao descobrir sobre ser portador do vírus.

0 comparecimento às consultas agendadas permite a continuidade da assistência, a prevenção de possíveis complicações e uma maior assiduidade ao tratamento. A maioria referiu comparecer 
às consultas, porém alguns relataram dificuldade no transporte, uma vez que nem sempre conseguiam essa garantia, pelo município (SILVA et al., 2015). Essa realidade precisa ser encarada pelo poder público como uma ação prioritária para que o paciente possa ter o direito à saúde assegurado com integralidade e equidade.

Quanto aos esquemas terapêuticos, faz-se importante ressaltar que inicialmente, estes eram complexos e as reações adversas frequentes, fatores esses que levavam os pacientes, muitas vezes ao abandono do tratamento. Atualmente, a adoção do esquema terapêutico combinado três em um, recomendado pelo MS em 2013, trouxe mudanças relevantes no sentido de simplificar o tratamento, reduzir as reações adversas aos medicamentos e ampliar a adesão (BRASIL, 2015). Nesta pesquisa, as queixas mais relatadas com o uso TARV foram: diarreia, náuseas e vômito. No entanto, a maioria dos participantes negou o surgimento das reações adversas com adoção da nova terapia, sendo esta considerada padrão ouro pelo MS. Esse fato é de extrema relevância pois espera- se que um maior número de pessoas se aglutine em favor do tratamento e reduza o índice de resistência e abandono à terapêutica.

\section{CONCLUSÃO}

Os achados deste estudo permitiram evidenciar que em relação aos aspectos sóciodemográficos e econômicos das PVHA houve uma predominância na população masculina, jovem, com baixa escolaridade e precário poder aquisitivo. Em se tratando dos fatores epidemiológicos e comportamentais, a maioria dos participantes do estudo adquiriu o HIV por transmissão sexual, referiu ser heterossexual, negou ter parceiro fixo e referiu não fazer uso de bebida alcoólica e tabagismo.

Quanto aos aspectos atinentes à internação hospitalar das pessoas vivendo com HIV/AIDS mais prevalentes foram: diagnóstico tardio do HIV com apresentação de algumas doenças relacionadas à AIDS (pneumonia, tuberculose e toxoplasmose cerebral) e os dados clínicos apresentados na internação foram: fraqueza, diarreia, febre e anemia.

\section{REFERÊNCIAS}

BARROS, S.G; SILVA, L.M. A terapia antirretroviral combinada, a política de controle da Aids e as transformações do Espaço Aids no Brasil dos anos 1990. Saúde debate, v. 41, n. esp. 3, p. 114-128, 2017.

BRASIL. Ministério da Saúde. Adesão ao tratamento antirretroviral no Brasil: Coletânea de

Estudos do Projeto ATAR. MS. Brasília, 2010. Disponível em:

<http:// www.AIDS.gov.br/publicacao/2015> Acesso em: 08 jun. 2016. 
BRASIL. Ministério da Saúde. Boletim epidemiológico HIVAIDS. MS. Brasília, 2016. Disponível em:<http://www.AIDS.gov.br/publicacao/2016> Acesso em: 02 mai. 2017.

BRASIL. Ministério da Saúde. Lei no 9.313 de13 de novembro de 1996. Dispõe sobre a Distribuição Gratuita de Medicamentos aos Portadores do HIV e Doentes de AIDS. Diário Oficial da União. Brasília. 1996; 14 nov. 1996.

BRASIL. Ministério da Saúde.Nota Informativa007-Protocolo de uso ARV2017. MS. Brasília, 2017. Disponível em: <http://www.azt.AIDS.gov.br/.../Nota\%20Informativa\%20007\%20 -20protocolo\%20de\%20uso\%2> Acesso em 28 jul. 2017.

BRASIL. Ministério da Saúde. Protocolo clínico e diretrizes terapêuticas para o manejo da infecção pelo HIV em adultos. MS. Brasília, 2015. Disponível em: < http:// www.AIDS.gov.br/> Acesso em: 27 Jun.16

BRASIL. Ministério da Saúde. Secretaria de Vigilância em Saúde. Programa Nacional de DST e Aids. Recomendações para terapia Antirretroviral em Adultos e Adolescentes Infectados pelo HIV. MS. Brasília, 2008.

FIGUEIREDO, R.M. et al. Adesão à terapia antirretroviral para HIV/AIDS. Rev Esc Enferm USP, v. 40, n. 4, p. 576-81, 2006.

GRECO, D. B. Trinta anos de enfrentamento à epidemia da Aids no Brasil, 1985-2015. Ciên Saúde Col, v. 21, n. 5, p. 1553-1564, 2016.

GREENACRE, M.; BLASIUS, J. Multiple correspondence analysis and related methods. New York: Chapman and Hall/CRC, 2006.

HIPOLITO, R. L. et al. Quality of life of people living with HIV/AIDS: temporal, socio-demographic and perceived health relationship. Rev Lat-Am Enferm, v. 25, e 2874, 2017.

KUMAR, A. et al. Profile of hospitalized HIV-Infected persons in the highly active Antiretroviral Therapy Era in Barbados. J Int Assoc Physicians Aids Care, v.6, n.2, p. 101-105, 2007.

MELCHIOR, R. et al. Desafios da adesão ao tratamento de pessoas vivendo com HIV/Aids no Brasil. Rev Saúde Públ, v. 41, n. 2, p. 87-93, 2007.

MÉRICI, A. Sergipe tem 4.943 casos notificados de AIDS. Secretaria de Estado da Saúde. Aracaju. 2016.Disponível em: <www.saude.se.gov.br/.../sergipe-tem-4-943-casos-notificados-AIDS-faixaetaria-de-30-a-39-a> Acesso em: 22 ago. 2017. 
NUNES, A. A. et al. Análise do perfil de pacientes com HIV/Aids hospitalizados após introdução da terapia antirretroviral (HAART). Ciên Saúde Col, v. 20, n. 10, p. 3191-3198, 2015.

PEDROSA, N.L. et al. Assistência especializada para pessoas com AIDS no estado do Ceará, Brasil. Rev Saúde Públ, v. 49, n. 77, p. 1-8, 2015.

ROMEU, G.A. et al. Avaliação da Adesão a Terapia Antirretroviral de Pacientes portadores de HIV. Rev Bras Farm Hosp Serv Saúde, v. 3, n. 1, p. 37-41, 2012.

SILVA, J. A. G. et al. Fatores associados à não adesão aos antirretrovirais em adultos com AIDS nos seis primeiros meses da terapia em Salvador, Bahia, Brasil. 2015. Cad Saúde Públ, v. 31, n. 6, p. 1188-1198, 2015.

SILVA, A.M. et al. Plano Diretor Estratégico do Hospital Universitário da Universidade Federal de Sergipe:Vencendo desafios com foco em melhores resultados para os usuários. EBSERH. Aracaju. 2016. Disponível em:

<http://www2.ebserh.gov.br/documents/16756/0/2016_03_02PDE20162017/ca0556d3-fb324d04-b8d2-75d192ccc6b3> Acesso em: 20 ago. 2017.

UNAIDS - United Nations Programme on HIV/AIDS. Novo Relatório do UNAIDS. Casa da ONU. Brasília, 2016. Disponível em: <https://unAIDS.org.br/2016/.../novo-relatorio-do-unAIDS-mostraque-182-milhoes-de-p> Acesso em: 13 ago. 2017. 
Recebido em: 12 de Fevereiro de 2019

Avaliado em: 13 de Abril de 2019

Aceito em: 13 de Abril de 2019

1 Mestre em Enfermagem pela Universidade Federal de Sergipe. Enfermeira do Hospital Universitário de Sergipe, Núcleo de Educação Permanente, Empresa Brasileira de Serviços Hospitalares (EBSERH). Email: cicera.silva@ ebserh.gov.br

2 Doutora em Ciências da Saúde pela Universidade Federal de Sergipe. Docente da Universidade Federal de Sergipe, Programa de Pós-graduação em Enfermagem, Departamento de Enfermagem. Email: ligiadolce@gmail.com

3 Mestre em Saúde Pública pela Universidade Estadual da Paraíba, Enfermeira Assistencial do Hospital Universitário de Sergipe. E-mail: julimusse@hotmail.com

4 Mestre em Enfermagem pela Universidade Federal de Sergipe, Enfermeira Assistencial do Hospital Universitário de Sergipe.Email: lidi_lima88@hotmail.com

5 Mestre em Enfermagem pela Universidade Federal de Sergipe, Enfermeira Assistencial do Hospital Universitário de Sergipe. Email: josiciat@hotmail.com

6 Doutora em Ciências Médicas pela Universidade de São Paulo. Docente da Universidade Federal de Sergipe, Programa de Pós-graduação em Enfermagem, Departamento de Enfermagem. Email: alziranery@uol.com.br

\section{可以

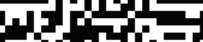

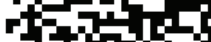

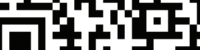

A autenticidade desse artigo pode ser conferida no site https://periodicos. set.edu.br

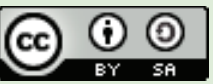

Este artigo é licenciado na modalidade acesso abertosob a Atribuição-Compartilhalgual CC BY-SA

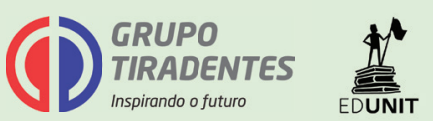


